\title{
Weight reduction improves markers of hepatic function and insulin resistance in type- 2 diabetic patients with non-alcoholic fatty liver
}

\author{
Al-Jiffri $\mathrm{O}^{1}$, Al-Sharif $\mathrm{FM}^{1}, *$ Abd El-Kader $\mathrm{SM}^{2}$, Ashmawy EM ${ }^{2}$
}

1. Department of Medical Laboratory Technology, Faculty of Applied Medical Sciences, King Abdulaziz University, Saudi Arabia.

2. Department of Physical Therapy, Faculty of Applied Medical Sciences, King Abdulaziz University, Saudi Arabia.

\begin{abstract}
Objective: The incidence of non-alcoholic fatty liver disease (NAFLD) is increasing dramatically affecting up to $30 \%$ of the population worldwide. At present, treatment options are limited and pharmacological management of NAFLD has had disappointing results. Some of the best available evidence to improve NAFLD concerns lifestyle modification.

Objective: To detect the degree of weight reduction needed to improve the markers of hepatic function and insulin resistance in type-2 diabetics with NAFLD.

Methods: One hundred type-2 diabetic male patients with NAFLD were included into this study and divided into two equal groups. Group (A) received aerobic exercise training in addition to diet regimen. Group (B) received no treatment intervention.

Results: There was a 26.99\%, 40.8\%, 33.81\%, 32.73\%, 37.8\% and 15\% reduction in mean values of Alkaline Phosphatase (ALP), Alanine Aminotransferase (ALT), Aspartate Aminotransferase (AST), Gamma - Glutamyltransferase (GGT) and Homeostasis Model Assessment-Insulin Resistance- index (HOMA-IR) and BMI respectively in group (A) at the end of the study. While there were significant differences between mean levels of the investigated parameters in group (A) and group (B) after treatment.

Conclusion: About $15 \%$ reduction in BMI is effective to improve the liver condition and insulin resistance in type-2 diabetics with NAFLD.

Key words: Non-alcoholic fatty liver, markers of hepatic function, insulin resistance, type-2 diabetes, weight reduction. African Health Sciences 2013; 13(3): 667 - 672 http:/ /dx.doi.org/10.4314/ahs.v13i3.21
\end{abstract}

\section{Introduction}

Nonalcoholic fatty liver disease (NAFLD) is an increasing condition associated with the sedentary lifestyle and poor nutritional habits, with high prevalence all around the World ${ }^{1}$. NAFLD is considered the hepatic manifestation of the metabolic syndrome, a cluster of metabolic abnormalities related to insulin resistance, including central obesity and dyslipidemia ${ }^{2}$. The imbalance in the metabolism of fatty acids in conjunction with the adipose tissue, liver tissue and systemic inflammation, are key factors for the development of insulin resistance, dyslipidemia and other cardiovascular risk factors

\begin{tabular}{|l|}
\hline *Corresponding author: \\
Prof. Shehab M. Abd El- Kader \\
Faculty of Applied Medical Sciences \\
Department of Physical Therapy \\
King Abdulaziz University \\
P.O. Box 80324, \\
Jeddah, 21589,Saudi Arabia. \\
Phone: +966-569849276 \\
E. mail: salmuzain@kau.edu.sa \\
\hline
\end{tabular}

African Health Sciences Vol 13 Issue 3 September 2013 associated with the 'nonalcoholic fatty liver disease' $(\mathrm{NAFLD})^{3}$.

Type 2 diabetes mellitus (T2DM) and obesity are the two metabolic conditions more closely associated with NAFLD and its progression towards advanced liver disease ${ }^{4}$. Paralleling the increasing prevalence of obesity, NAFLD has become common and potentially serious ${ }^{5}$.They both stem from alterations in insulin action (insulin resistance), a metabolic state in which physiologic concentrations of insulin produce a lower-thannormal biologic response, or higher-than-normal insulin concentrations are necessary to elicit a normal metabolic response. Thus, any measure able to modify overweight/obesity or a sedentary lifestyle, as well as the associated $\operatorname{defect}(\mathrm{s})$ in insulin action represents both a preventive measure and a treatment option for NAFLD ${ }^{6}$.

There is no proven treatment for patients with NAFLD are currently available. Weight reduction and lifestyle modifications with diet changes and increased physical activity are usually 
recommended as the first step in the treatment of patients with this condition. Achieving and maintaining weight reduction may improve NAFLD, but the results of several reports are inconsistent ${ }^{7}$. Studies reporting the effect of weight reduction in NAFLD to date have included small numbers of patients that were treated for a short period of time ${ }^{8}$.

The purpose of this study was to investigate the degree of weight reduction needed to improve the markers of hepatic function and insulin resistance in type-2 diabetic patients with NAFLD.

\section{Methods \\ Subjects}

One hundred type-2 diabetic male patients with NAFLD with body mass index (BMI) ranged from 30 to $35 \mathrm{Kg} / \mathrm{m}^{2}$, their age ranged from 35 to 55 years, type 2 diabetic patients were selected according to the criteria published by the American Diabetes Association 9, free from other liver, metabolic or genetic diseases. Participants were included in this randomized controlled study and divided into two equal groups; group (A) received physical training combined with dietary measures. The second group (B) received dietary measures only.

Participants were identified from a large number of patients attending the Liver Clinic in King Abdulaziz University Teaching Hospital, with a histological diagnosis of NAFLD. The diagnosis of NAFLD was based on the following criteria: (1) elevated aminotransferases (AST and/or ALT); (2) liver biopsy showing steatosis in at least $10 \%$ of hepatocytes; and (3) appropriate exclusion of liver disease of other aetiology including alcohol- or druginduced liver disease, autoimmune or viral hepatitis, cholestatic or metabolic/genetic liver disease. These other liver diseases were excluded using specific clinical, biochemical, serologic tests radiographic and/ or histological criteria.

Exclusion criteria included: smoking; hypertension, personal history of cardiovascular diseases, thyroid disease and orthopedic problems inhibiting treadmill training. This study was approved by the Scientific Research Ethical Committee, Faculty of Applied Sciences, King Abdulaziz University. Informed consent was obtained from all participants. All participants were free to withdraw from the study at any time. If any adverse effects had occurred, the experiment would have been stopped, with this being announced to the Human Subjects Review Board.

\section{Chemical analysis}

Blood samples were collected from the antecubital vein at the beginning and end of the treatment program. Subjects had blood drawn at the same time in the morning on each occasion (between 8 and $10 \mathrm{AM}$ ). Subjects lay supine for $10 \mathrm{~min}$ prior to the blood collection. $10 \mathrm{ml}$ of blood was drawn drawn into a tube containing few milliliters of sodium citrate; plasma was separated from the blood by centrifugation $(120 \times \mathrm{g}$ for $15 \mathrm{~min})$ at room temperature. Markers of hepatic function (aspartate aminotransferase, AST; alanine aminotransferase, ALT; alkaline phosphatase, ALP and Homeostasis Model Assessment-Insulin Resistance (HOMA) index for insulin sensitivity was computed following this equation: [fasting glycemia ( $\mathrm{mmol} / \mathrm{L}) \mathrm{X}$ fasting insulin $(\mathrm{mIU} / \mathrm{L})] / 22.5^{10}$ were measured by the colorimetric enzymatic method using an automatic spectrophotometer and respective kits for analysis (Bioclin, Quibasa, Belo Horizonte, MG, Brazil). Human insulin was measured with an insulin kit (Roche Diagnostics, Indianapolis, IN, USA) using a cobas immunoassay analyzer (Roche Diagnostics). All samples were assayed in duplicate, and the mean of the paired results was determined.

\section{Evaluation of anthropometric parameters}

All measurements were performed at pretreatment and after three months at the end of the study. The participants were measured whilst wearing their undergarments and hospital gowns. Height was measured with a digital stadiometer to the nearest $0.1 \mathrm{~cm}$ (JENIX DS 102, Dongsang, South Korea). Body weight was measured on a calibrated balance scale to the nearest $0.1 \mathrm{~kg}$ (HC4211, Cas Korea, South Korea), and BMI was calculated as BMI = Body weight / (Height) ${ }^{2}$.

\section{The physical training program}

The aerobic treadmill-based training program (PRECOR 9.1/ 9.2, China) was set to $65 \%-75 \%$ of the maximum heart rate (HRmax) according to a modified Bruce protocol. This rate was defined as the training heart rate (THR).After an initial, 5-minute warm-up phase performed on the treadmill at a low load, each endurance training session lasted 30 minutes and ended with 5-minute recovery and relaxation phase. All patients performed three weekly sessions (i.e. a total of 36 sessions per patient over a 3-month period). 
The prescribed low calorie diet

The interview-based food survey was performed for all patients by dieticians to specify previous food habits and possible anomalies in dietary behavior. The prescribed low calorie diet was balanced, with $15 \%$ as protein, 30 to $35 \%$ as fat and 50 to $55 \%$ as carbohydrate, on average, in order to provide about 1200 Kilocalories daily for two months for whole participants in this study.

\section{Statistical analysis}

The mean values of ALP, ALT, AST, GGT, HOMAIR and BMI obtained before and after three months in both groups were compared using paired " $\mathrm{t}$ " test. Independent " $t$ " test was used for the comparison between the two groups $(\mathrm{P}<0.05)$.

\section{Results}

This study was an attempt to investigate the degree of weight reduction (a result that is needed to improve the markers of hepatic function and insulin resistance in type-2 diabetic patients with NAFLD. However, no adverse effects occurred as the diet regimen was low caloric diet not a very low caloric diet and the aerobic exercise training was of moderate intensity ranged from $65 \%-75 \%$ of HRmax, so the data of all the participants were available for analysis. There was a $26.99 \%, 40.8 \%$, $33.81 \%, 32.73 \%, 37.8 \%$ and $15 \%$ reduction in mean values of ALP, ALT, AST, GGT, HOMA-IR and BMI respectively in group (A) at the end of the study. The mean values of ALP, ALT, AST, GGT, HOMAIR and BMI were significantly decreased in group received aerobic exercise training in addition to diet regimen. While the results of group (B) received no treatment intervention were not significant. Also, there were significant differences between mean levels of the investigated parameters in group (A) and group (B) after treatment (table 1, 2, 3) $(\mathrm{P}<0.05)$.

Table 1: Mean value and significance of the pre and post test values of ALP, ALT, AST, GGT, HOMA-IR and BMI in the training group

\begin{tabular}{|c|c|c|c|c|}
\hline & \multicolumn{2}{|c|}{ Mean + SD } & \multirow[t]{2}{*}{ t- value } & \multirow[t]{2}{*}{ P-value } \\
\hline & Pre & Post & & \\
\hline$\overline{\operatorname{ALP}(\mathrm{U} / \mathrm{L})}$ & $74.56 \pm 9.32$ & $58.71 \pm 8.26$ & 9.23 & 0.0015 \\
\hline ALT (U/L) & $46.88 \pm 5.41$ & $33.28 \pm 4.76$ & 8.64 & 0.0013 \\
\hline AST (U/L) & $45.98 \pm 6.63$ & $34.36 \pm 5.11$ & 9.15 & 0.0027 \\
\hline GGT(U/L) & $28.63 \pm 3.26$ & $21.57 \pm 3.1$ & 7.34 & 0.0056 \\
\hline HOMA-IR & $4.92 \pm 2.78$ & $3.57 \pm 1.3$ & 6.12 & 0.0083 \\
\hline$\underline{\mathrm{BMI}}\left(\mathrm{Kg} / \mathrm{m}^{2}\right)$ & $32.11 \pm 3.54$ & $27.25 \pm 2.68$ & 8.33 & 0.0094 \\
\hline
\end{tabular}

ALT $=$ Alanine aminotransferase

GGT $=$ Gamma - glutamyltransferase

AST $=$ Aspartate aminotransferase $\quad$ ALP $=$ Alkaline phosphatase $\quad B M I=$ Body mass index

HOMA-IR $=$ Homeostasis Model Assessment-Insulin Resistance (HOMA-IR) index

Table 2: Mean value and significance of the pre and post test values of ALP, ALT, AST, GGT, HOMA-IR and BMI in the control group

\begin{tabular}{lcccc}
\hline & \multicolumn{1}{c}{ Mean + SD } & t- value & P-value \\
\cline { 2 - 3 } & Pre & Post & & \\
\hline ALP (U/L) & $73.31 \pm 8.92$ & $73.68 \pm 9.23$ & 0.76 & 0.139 \\
ALT (U/L) & $47.22 \pm 6.05$ & $47.91 \pm 6.75$ & 0.85 & 0.144 \\
AST (U/L) & $46.16 \pm 6.87$ & $46.87 \pm 7.24$ & 0.49 & 0.172 \\
GGT(U/L) & $29.33 \pm 3.76$ & $30.01 \pm 3.65$ & 0.91 & 0.115 \\
HOMA-IR & $4.98 \pm 2.51$ & $5.13 \pm 2.44$ & 0.56 & 0.161 \\
BMI & $32.37 \pm 3.92$ & $32.64 \pm 4.26$ & 0.36 & 0.182 \\
\hline ALT = Alanine aminotransferase & GGT = Gamma - glutamyltransferase \\
AST = Aspartate aminotransferase & ALP = Alkaline phosphatase & BMI= Body mass index \\
HOMA-IR = Homeostasis Model Assessment-Insulin Resistance (HOMA-IR) index
\end{tabular}


Table 3: Mean value and significance of the post test values of ALP, ALT, AST, GGT, HOMA-IR and BMI in the training and control groups

\begin{tabular}{lclll}
\hline & \multicolumn{2}{c}{ Mean + SD } & t- value & P-value \\
& Training group & Control group & & \\
\hline ALP (U/L) & $58.71 \pm 8.26$ & $73.68 \pm 9.23$ & 7.86 & 0.0062 \\
ALT (U/L) & $33.28 \pm 4.76$ & $47.91 \pm 6.75$ & 6.22 & 0.0074 \\
AST (U/L) & $34.36 \pm 5.11$ & $46.87 \pm 7.24$ & 7.07 & 0.0085 \\
GGT(U/L) & $21.57 \pm 3.18$ & $30.01 \pm 3.65$ & 5.93 & 0.0073 \\
HOMA-IR & $2.64 \pm 1.37$ & $5.13 \pm 2.44$ & 4.75 & 0.0091 \\
BMI & $27.25 \pm 2.68$ & $32.64 \pm 4.26$ & 6.12 & 0.0088 \\
\hline
\end{tabular}

ALT $=$ Alanine aminotransferase $\quad$ GGT $=$ Gamma - glutamyltransferase

AST $=$ Aspartate aminotransferase $\quad$ ALP $=$ Alkaline phosphatase $\quad B M I=$ Body mass index

HOMA-IR = Homeostasis Model Assessment-Insulin Resistance (HOMA-IR) index

\section{Discussion}

As the degree and rate of weight reduction needed to improve the liver condition in patients with NAFLD remains unknown. The aim of this study was to detect the impact of weight reduction on liver enzymes and insulin resistance in type-2 diabetic patients with NAFLD received aerobic exercise training in addition to diet regimen. Based on our findings, a $15 \%$ reduction in BMI and body weight could be recommended as an initial therapeutic target to improve the liver condition and insulin resistance in type-2 diabetic patients with NAFLD. However, the oral hypoglycemic agents who were received by participants in both groups may have some effect on insulin resistance, the more profound improvement in insulin resistance in group (A) and the little non significant changes in group (B) proved that weight reduction was of great value to enhance the therapeutic pharmacological effects of oral hypoglycemic agents. Results of this study supported with many previous studies ${ }^{11-21}$.

Adherence to combined dietary restriction and increased physical activity result in larger and progressive weight loss that can be maintained through the years ${ }^{11}$. Physical activity has an independent and beneficial effect on fatty liver ${ }^{12}$. Physical exercise promotes both weight loss, by increasing the negative energy balance, and insulin sensitivity, the determinant of fatty liver, by promoting triglyceride consumption in the skeletal muscle tissue ${ }^{13}$.Maintaining or increasing physical activity provides health benefits for patients with fatty liver, independent of changes in weight ${ }^{14}$.

Weight, blood biochemical data as aminotransferase, albumin, cholinesterase, total cholesterol and fasting blood glucose values were significantly decreased after a program of restricted diet and exercise (walking or jogging) for a trial period of 3 months in a previous study on twentyfive obese patients with fatty liver ${ }^{15}$.

A 10-week diet-plus-exercise and exerciseonly therapeutic lifestyle programs are effective for improving anthropometric indices, insulin sensitivity, ultrasound findings and physical fitness in ultrasounddiagnosed NAFLD patients. However, the range of improvement in patients on the diet-plus-exercise program is more obvious than that in patients on the exercise-only program. Moreover, the diet-plusexercise program resulted in significant improvement in liver biochemistry, but the exercise-only program did not. In summary, diet plus exercise is more efficacious than exercise alone in the lifestyle modification treatment of NAFLD ${ }^{16}$.

In general, weight loss was shown to improve liver histology ${ }^{17}$, whereas gradual weight reduction and increased physical activity improves liver enzymes ${ }^{15,18}$, insulin sensitivity and quality of life ${ }^{19}$. In T2DM patients, moderate weight reduction $(8 \%)$ was shown to reduce liver fat that was accompanied by a dramatic improvement in hepatic insulin resistance, with return to normal suppression of hepatic glucose production by insulin ${ }^{20}$.

Regular exercise was also associated with significantly greater ALT improvement and normalization. For instance, exercise is known to improve the sensitivity of muscle mass to insulin ${ }^{21}$. Furthermore, exercise that was not enough to reduce body weight showed modest therapeutic effect in reducing visceral fat and improving glucose intolerance 22 .

Energy restriction resulting in even modest weight loss suppresses endogenous cholesterol synthesis which leads to a decline in circulating lipid 
concentrations and increased insulin sensitivity that contributes in improving lipoprotein profile after treatment of obesity ${ }^{23,24}$. Low carbohydrate diet and a low fat-diet may be useful in reducing the intrahepatic triglyceride content ${ }^{25,26}$.Furthermore, $5 \%$ or greater weight reduction was associated with decrease in serum triglycerides, increase in serum HDL-cholesterol and decrease in fasting blood glucose. This indicates that achieving and maintaining $5 \%$ weight reduction will improve not only the liver condition but also several other components of the metabolic (insulin resistance) syndrome ${ }^{18}$.

\section{Conclusion}

Based on our findings, a $15 \%$ reduction in BMI is effective to improve the liver condition and insulin resistance in type-2 diabetic patients with NAFLD to improve the liver condition.

\section{Acknowledgment}

This project was funded by the Deanship of Scientific Research (DSR), King Abdulaziz University, Jeddah, under grant no. (49/142/1432). The authors, therefore, acknowledge with thanks DSR technical and financial support.

\section{References}

1. Fan J, Farrell G. Epidemiology of nonalcoholic fatty liver disease in China. J Hepatol 2009; 50: 204-210.

2. Angulo P. Nonalcoholic fatty liver disease. $N$ Engl J Med 2002; 346: 1221-1231.

3. Fabbrini E, Sullivan S, Klein S. Obesity and nonalcoholic fatty liver disease: biochemical, metabolic, and clinical implications. Hepatology 2010; 51: 679-689.

4. Marchesini G, Bugianesi E, Forlani G. Nonalcoholic fatty liver, steatohepatitis, and the metabolic syndrome. Hepatology 2003; 37: $917-$ 923.

5. Ito O, Muroya Y, Mori N, Nagasaka M, Kanazawa M, Kohzuki M. Poster 232: Improvement of Pediatric Nonalcoholic Fatty Liver Disease by Diet and Physical Exercise: A Case Report. Arch Phys Med Rehabil 2007; 88 (9): E76

6. Targher G, Bellis A, Fornengo P, Ciaravella F, Pichiri I, Perin P, etal. Prevention and treatment of nonalcoholic fatty liver disease. Dig Liver Dis 2010; 42(5): 331-340.
7. Andersen T, Gluud C, Franzmann M , Christoffersen P. Hepatic effects of dietary weight loss in morbidly obese subjects. J Hepatol 1991; 12: 224-229.

8. Wang R, Koretz R, Yee H. Is weight reduction an effective therapy for nonalcoholic fatty liver? A systematic review. Am J Med 2003; 115: 554 559.

9. American Diabetes Association. Diagnosis and classification of diabetes mellitus. Diabetes Care 2008;31(Suppl. 1):S55-60.

10. Wilund KR. Is the anti-inflammatory effect of regular exercise responsible for reduced cardiovascular disease? Clin Sci (Lond) 2007; 112(11):543-55.

11. Saris W, Blair S, van Baak M. How much physical activity is enough to prevent unhealthy weight gain? Outcome of the IASO 1st Stock Conference and consensus statement. Obes Rev 2003; 4:101-114.

12. Cox K, Burke V, Morton A. Independent and additive effects of energy restriction and exercise on glucose and insulin concentrations in sedentary overweight men. Am J Clin Nutr 2004; 80: 308-316.

13. Kantartzis K, Thamer C, Peter A. High cardiorespiratory fitness is an independent predictor of the reduction in liver fat during a lifestyle intervention in non-alcoholic fatty liver disease. Gut 2009; 58: 1281-1288.

14. St George A, Bauman A, Johnston A. Independent effects of physical activity in patients with nonalcoholic fatty liver disease. Hepatology 2009; 50: 68-76.

15. Ueno T, Sugawara H, Sujaku K, Hashimoto O, Tsuji R, Tamaki S, etal. Therapeutic effects of restricted diet and exercise in obese patients with fatty liver. Journal of Hepatology. 1997; 27, (1):103-107.

16. Chen S, Liu C, Li S, Huang H, Tsai C, Jou H . Effects of Therapeutic Lifestyle Program on Ultrasound-diagnosed Nonalcoholic Fatty Liver Disease. Journal of the Chinese Medical Association 2008; 71(11):551-558.

17. Tilg H, Kaser A. Treatment strategies in nonalcoholic fatty liver disease. Nat Clin Pract Gastroenterol Hepatol 2005; 2:148-155.

18. Suzuki A, Lindor K, St Saver J, Lymp J, Mendes F, Muto A, Okada T, Angulo P. Effect of changes on body weight and lifestyle in nonalcoholic fatty liver disease. Journal of Hepatology 2005; 43(6):1060-1066 
19. Hickman I, Jonsson J, Prins J . Modest weight loss and physical activity in overweight patients with chronic liver disease results in sustained improvements in alanine aminotransferase, fasting insulin, and quality of life. Gut 2004; 53: 413-419.

20. Petersen K, Dufour S, Befroy D. Reversal of nonalcoholic hepatic steatosis, hepatic insulin resistance, and hyperglycemia by moderate weight reduction in patients with type 2 diabetes. Diabetes 2005; 54: 603-608.

21. Perseghin G, Price T, Petersen K, Roden M, Cline $G$, Gerow K. Increased glucose transport-phosphorylation and muscle glycogen synthesis after exercise training in insulin-resistant subjects. N Engl J Med 1996; 335: 1357-1362.

22. Ross R, Dagnone D, Jones P, Smith H, Paddags A, Hudson R. Reduction in obesity and related comorbid conditions after diet-induced weight loss or exercise-induced weight loss in men. A randomized controlled trial. Ann Intern Med 2000; 133: 92-103.
23. Di Buono, M, Hannah JS, Katzel LL, Jones PJ. Weight loss due to energy restriction suppresses cholesterol biosynthesis in overweight, mildly hypercholesterolemic men. J. Nutr 1999; 129 (8): $1545-8$.

24. Lamarche B, Despress J, Pouliot MC, Moorjani S, Lupien P, Jheriault G, Tremblay A, Nadeau A, Bouchard C. Is body fat loss a determinant factor in the improvement of carbohydrate and lipid metabolism following aerobic exercise training in obese women?. Metabolism 1992; 41 (11): 1249-1256.

25. Browning J, Davis J, Saboorian M. A lowcarbohydrate diet rapidly and dramatically reduces intrahepatic triglyceride content. Hepatology 2006; 44: 487-488.

26. Weaterbacka J, Lammi K, Hakkinen A. Dietary fat content modifies liver fat in overweight nondiabetic subjects. J Clin Endocrinol Metab 2005; 90:2804-2809. 